\title{
Dronedarone: Who, When, Why And How Should It Be Prescribed And Monitored?
}

Misfir Theeb Alqahtani*

King Salman Hospital, Pharmacy department, Aysha Street, Riyadh, Saudi Arabia

*Corresponding author: Misfir Theeb Alqahtani, King Salman Hospital, Pharmacy department, Aysha street, Riyadh, Saudi Arabia, Tel: 00966555388894; E-mail: Misfir_qh@hotmail.com

Received date: June 18, 2015; Accepted date: July 10, 2015; Published date: July 15, 2015

Copyright: (C) 2015 Alqahtani MT. This is an open-access article distributed under the terms of the Creative Commons Attribution License, which permits unrestricted use, distribution, and reproduction in any medium, provided the original author and source are credited.

\section{Commentry}

Dronedarone is defined as "an oral anti-arrhythmic drug (ADD), indicated for clinically stable patients with a history of non-permanent atrial fibrillation (AF), for preventing recurrence of $\mathrm{AF}$, or reducing ventricular rate (VR) [1]." It is "a non-iodinated benzofuran derivative of amiodarone".

It potentially blocks multiple ion currents, "including the rapidly activating delayed-rectifier potassium current, the slowly activating delayed-rectifier potassium current, the inward rectifier potassium current, the acetylcholine activated potassium current, peak sodium current, and L-type calcium current, and exhibits antiadrenergic effects [2]." It is also used for the maintenance of sinus rhythm and ventricular response control during atrial fibrillation episodes [2].

It is utilized to treat abnormal heart rhythm in patients with an abnormal heart rhythm (i.e. Atrial fibrillation or atrial flutter) lasting for 6 months. It helps reduce the risk of hospitalization secondary to heart problems [3].

\section{Who Should Take Dronedarone?}

It is mostly used by patients who are part of the elderly population who are also in moderate to high-risk. It was deemed unsafe in the case of a controlled trial in patients who were hospitalized due to symptomatic heart failure and severe left ventricular systolic dysfunction whose treatment was prematurely terminated because of worsening heart failure or even death with dronedarone use. In randomized trials, however, dronedarone showed more potential to be effective than placebo at reducing AF recurrence [1].

\section{When Is Dronedarone To Be Taken?}

Dronedarone is suggested to be taken in $400 \mathrm{mg}$ dose, twice a day together with meals, for a complete cycle of treatment as prescribed by a physician. It is well-tolerated most of the time but there were reports claiming the following adverse effects: diarrhea, nausea and vomiting, and abdominal pain [1].

\section{Why Should Dronedarone Be Prescribed?}

According to the Regional Drug and Therapeutics Centre, the drug shows to be more efficient in comparison with placebo in randomized trials at reducing the $\mathrm{AF}$ recurrence, but amiodarone is a more effective choice. In contrast, dronedarone results to lesser adverse effects versus amiodarone. In fact, dronedarone is advised as a secondline treatment alternative for AF for patients who have cardiovascular risks [1].
Its effectiveness in maintaining sinus rhythm and in controlling VR during recurrences of AF has been compared with placebo [1]. FDA warns physicians from prescribing dronedarone to patients with permanent atrial fibrillation. In January of 2011, the FDA issues a Drug Safety Communication in certain rare cases of serious liver complications seen in patients [3].

\section{How Dronedarone Should Be Prescribed And Monitored?}

After new evidence of risk factors related to the use of dronedarone was found, there were cardiovascular, hepatic, ad pulmonary risks that should be monitored. Although the benefits of its treatment is said to outweigh its risks, it is advised to be prescribed as a last resort after all other treatment options have been analysed [4]

In order to practice safe administration, prescription and support monitoring, patients are encouraged to have a review of their treatment on the next scheduled appointment to guarantee that they are still eligible for treatment. Also, regular monitoring of cardiac, liver, and renal functions during treatment is highly advised [4].

\section{Costing Comparison}

In comparison of three drugs by the Drug Tariff and eMIMS in March 2010, it revealed that dronedarone is more expensive than sotalol and amiodarone. The analysis was done for a 28 -days course of treatment, and it shows dronedarone, prescribed in $400 \mathrm{mg}$ dose to be taken twice a day (BD), costs $£ 63.00$; Sotalol $80 \mathrm{mg}$ twice a day costs $£ 1.91$; and Amiodarone $200 \mathrm{mg}$ taken once a day costs $£ 1.85$ [1].

\section{An Evidence-based Study on Dronedarone}

In one study, the researchers discovered that dronedarone greatly diminishes renal creatinine CL and NMN CL. However, it did not change renal sistrin $\mathrm{CL}, \mathrm{PAH} \mathrm{CL}$, and other renal parameters. The findings suggest that there is a particular partial inhibition of tubular organic cation transporters (OCT) as evidenced by its ability to reduce renal creatinine and NMN clearance by 18 percent. Therefore, a limited increase in serum creatinine should be expected in using dronedarone during treatment, but without a decline in renal function [5].

Another study found that dronedarone decreases the mortality and morbidity incidence in patients who are highly at risk for atrial fibrillation, but is deemed not safe for patients with severe heart failure [6]. It was also discovered that dronedarone reduced patient hospitalization attributed to cardiovascular problems or death in patients with atrial fibrillation [6]. 
Citation: Alqahtani MT (2015) Dronedarone: Who, When, Why And How Should It Be Prescribed And Monitored?. J Pharmacovigilance 3: 169. doi:10.4172/2329-6887.1000169

Page 2 of 2

Another study published in the Journal of Cardiovascular Electrophysiology deemed amiodarone to be the most effective drug, but also revealed that it possesses potential serious extra cardiac adverse effects. Dronedarone, since it lacks iodine moiety, decreases lipophilicity by decreasing the accumulation of tissue and shortened half-life. The researchers add that it similarly functions like amiodarone in terms of multichannel blocking effects, inhibiting transmembrane of Sodium, Potassium, and Calcium, as well as slow Ltype calcium channels, and its antiadrenergic rhythm-control characteristics, thus dronedarone is proven to be safe and effective [7].

In fact, some conditions, such as torsades de pointes - a severe side effect of amiodarone, are not yet proven to exist in patients with dronedarone. It is said to be because of dronedarone's limited effect on thyroid function and hormone levels in clinical trials both on animals and in humans [8].

\section{References:}

1. Regional Drug and Therapeutic Centre (2010) Dronedarone. New Drug Evaluation No. 103.
2. Patel C, Yan G, Kowey P (2009) Dronedarone, New Drugs and Technologies. Circulation 120: 636-644.

3. Multaq (dronedarone) Information, Drugs. US Food and Drug Administration (FDA).

4. Dronedarone (Multaq): cardiovascular, hepatic and pulmonary adverse events - new restrictions and monitoring requirements (2011) Drug Safety Update.

5. Tschuppert Y, Buclin T, Rothuizen L, Decosterd L, Galleyrand J, et al. (2007) Effect of dronedarone on renal function in healthy subjects. British Journal of Clinical Pharmacology 64: 785-791.

6. Hohnloser S, Crijins HJ, Eickels M, Gaudin C, Page RL, et al. (2009) Effect of Dronedarone on Cardiovascular Events in Atrial Fibrillation. New England Journal of Medicine 360: 360-678.

7. Wegener F, Ehrlich J, Hohnloser S (2006) Dronedarone: An Emerging Agent with Rhythm- and Rate-Controlling Effects. Journal of Cardiovascular Electrophysiology 17: s17-s20.

8. Kathofer S, Thomas D, Karle CA (2005) The Novel Antiarrhythmic Drug Dronedarone: Comparison with Amiodarone. Cardiovascular Drug Reviews 23: 217-230. 